\title{
Presenting a Model for Identifying the Best Location of Melli Bank ATMS by Combining Clustering Algorithms and Particle Optimization
}

\author{
Abdolhussein Shakibayinia \\ Department of Computer, Dezful Branch,Islamic \\ Azad University, Dezful,Iran
}

\author{
${ }^{*}$ Faraz Forootan \\ Department of Computer, Dezful Branch,Islamic \\ Azad University, Dezful,Iran
}

\begin{abstract}
The Interbank Information Exchange Network (Shetab or Acceleration) has started since 2002 and the purpose was integrating and connecting card systems of all banks in the country. Currently, the Acceleration Center has been acting as Melli bank card switch in the country, and all the banks in the country are its member. These operations cover a wide range of transactions, such as cash withdrawals, electronic purchases, fund transfers, paying bills and residual payments. Shetab center processes more than two and a half million transactions per day. At present, the amount of fees received from each network transaction is 500 to 22,000 Rial, which is considered as a fee for the client's bank as revenue and for the client bank. And it does not cost any expenses to the customer, thus banks are looking for earning revenue from this service.

In this, first the list of ATMs that Melli Bank pays them service fee are considered, then by using the clustering algorithm, locations were arranged for an ATM so Melli Bank pay less fee. In this study, the combination of three K-means algorithms and particle optimization algorithm and genetic algorithm were used. Davies-Bouldin Index was used to assess clustering.

Then, the proposed clustering along with another clustering algorithm was evaluated and it was shown that the proposed algorithm is performing better. 8 locations for ATM were presented in proposed clustering algorithm, which is the result of the proposed clustering.
\end{abstract}

Keywords: Particle optimization algorithm, Banking, ATM

\section{INTRODUCTION}

Today, the banking system consisting of the central bank and commercial banks play a decisive role in the economic development of the country. Banks are using the most advanced technology and needed tools and the most extensive international networks in the world, to be able, through a variety of computer services, make monetary deposits legal and legal persons at the best possible cost and at the lowest cost and with the most secure way, from one place to another, or from an account to another account as soon as possible [1].

The payment system is a mechanism that transfers money from an account in a bank to an account in another bank, thus the role of the paying system in the economy is like the veins that bring money to different economic firms, so the reality is that the accurate management and supervision and smoothly functioning of the payment system in the monetary part of the country is one of the main duties of central banking in the today world [2].

Shetab started in 2002 with the aim of integrating and linking card systems of all banks in the country. At present, the Acceleration Center has play a role as Melli bank card switch in the country, and all the banks in the country are its members. The operations cover a wide range of transactions, such as cash withdrawals, electronic purchases, fund transfers, paying bills and residual payments. Shetab processes more than two and a half million transactions per day. At present, the amount of fees received from each network transaction is 398 Tomans, which is for the bank as revenue and for the client's bank as a cost and does not create any costs for the client.
The advancement in communications science and modern banking technology has led the service system to faster delivery and more consistent services. Today, the level of access to these facilities is almost the same for all financial institutions and banks and banking services companies. In fact, the point that makes a bank superior to other financial institutions and banks is providing a distinct, fast and continuous service. ATM is one of the tools that can help customers accelerate automated tasks [3].

Automated Teller Machine (ATM) is a device that can be used for depositing and withdrawing from customer accounts, changing card and account statements, transferring funds, paying bills, account balance and some other services through a Melli card without the need for the operator. ATMs are the most prominent feature of the evolution of banking services based on modern technology. Based on the policies of the Melli Bank of Iran to develop the use of modern banking systems, since 1997, the installation of the first ATM series in the selected branches began, and so far, with the installation of more than 6,600 ATMs, the Melli Bank of Iran has the largest number of installed ATMs in among Banks in the country.

Locating new ATMs is very important for the organization due to its rapid support, costs and payment fees for the transaction of other bank cards (other than the Melli Bank), and making related decisions are very sensitive, important and worthwhile. This is the responsibility of senior officials and informatics supervised by the provincial branch office. For example, the salary paid to one of the ATMs of the Bank of SADERAT has been 434,225,087 Rials in 2015, thus to decide and choose the best location to other branches of the Melli Bank which are 
closer also for better maintenance. The more important places are reportedly by Accelerator Center, and the Melli Bank ATM card transactions have accepted the most competitive and large fees paid to them. To do this, we have chosen the western region of Ahwaz to explore and derive the final model, which will calculate the best location of the new ATM based on the geographical coordinates of ATMs of the Melli Bank and the rival [4].

In this paper, by combining the k-means clustering algorithms and particle optimization, with the criteria for increasing support and reducing out-of-service time and reducing payroll fees to other competing banks, we will give the best place to the senior executives of the bank. Gave Clustering is one of the most important issues of non-monitoring learning as well as the most common data mining techniques used to classify data sets into specific subsets. The k-means algorithm is also one of the most popular clustering algorithms with easy implementation and fast performance, but being sensitive to first cluster centers can only produce a local optimal response. By combining the k-means clustering method with the particle group optimization algorithm (due to the existence of decimal data), we improved the accuracy of clustering.

The study consists of two parts. In the first section, the proposed algorithm is presented and in the second section the results of the research are shown..

\section{THE PROPOSED ALGORITHM}

The proposed algorithm is presented in this section, which has two parts. In the first step, the K-means algorithm is used to select the start points of the K-means algorithm from the particle optimization algorithm, then in the second stage, heuristic algorithms are used to determine the start points of the particle algorithm and then the genetic algorithm is used to optimize the particle optimization algorithm.

\section{2-1. The proposed algorithm}

Two cases are considered to use ATM for the withdrawal of money. In the first case, the card that is taken from it is related to the same bank. In the latter case, the card is not from the same bank. In the first case, for example, the Melli Bank card withdrawn from the Melli Bank ATM, and in the latter case, for example, the Melli Bank withdraw money from the Tejarat bank ATM. In the latter case, due to the use of the Tejarat bank ATM, the Melli Bank should pay the Tejarat Bank.

In this research, it was tried that the Melli Bank's customers refer less to ATMs of other banks. To do this, the information about the number of Melli Bank's customers visiting has been removed from other banks and the amount has been deducted and the location of those banks has been analyzed and continue to add new ATMs for the customer of those places. The structure of the data used is shown in Table 1.
Table 1 ATMs

\begin{tabular}{|r|l|l|c|}
\hline \multicolumn{1}{|c|}{ Row } & \multicolumn{2}{|c|}{ Title } & Variable \\
\hline \multirow{2}{*}{1} & \multirow{2}{*}{ Features } & $\mathrm{X}$ & Discrete \\
\cline { 3 - 4 } & & $\mathrm{Y}$ & Discrete \\
\hline 2 & Referrals number & Discrete \\
\hline 3 & Withdrawn amount & Discrete \\
\hline
\end{tabular}

Weighted clustering is used in this data. The data structure is such that a number of parameters are considered as those data parameters, and a number of parameters are considered as data weights. Whatever the weight is given, the data is considered to more distant.

In the bank's data, the two parameters $\mathrm{X}$ and $\mathrm{Y}$ are considered as data parameters, and the number of referrals and the withdrawn amount is considered as the weight of the ATM. In the data, the more weighted it is the ATM is considered to be farther, so the parameters are reversed so that the number of visits and the amount removed are increased, near ATMs are considered in equation (1) the calculation of the points' weight is shown.

$W_{i}=\frac{1}{\text { number of referrals }}+\frac{1}{\text { the withdrawn amount }}$

In which, both the relationship between the number of referrals and the withdrawn amount are reversed and its total is considered as the weight of the bank.

In the key clustering algorithm, the parameter is a distance, with the difference that this distance is considered in the relation of the record weight. This calculation is shown in equation (2).

$d(i, j)=\sqrt{\left(w_{i} x_{i}-w_{j} x_{j}\right)^{2}+\left(w_{i} y_{i}-w_{j} y_{j}\right)^{2}}$

The difference between each parameter in the weight of the number of referrals and the amount of withdrawal is multiplied to determine its effect on the distance between ATMs.

The proposed algorithm consists of two first parts in the first phase of the particle optimization algorithm and the K-means algorithm are combined. In the second step, the heuristic algorithms are used to determine the starting points of the particle algorithm, and then the genetic algorithm is used to optimize the particle optimization algorithm.

\section{2-2. Problem solving by combining particle optimization and K-means algorithm}

The problem solving structure is that the genetic algorithm is responsible for determining the starting points of the K-Means algorithm. The K-Means algorithm depends on the starting points. If the starting points are well chosen, the output of the K-Means algorithm will be better, and if the points are not selected, then the output is not good in terms of clustering. The parameters $\mathrm{X}$ and $\mathrm{Y}$ are discrete and the particle optimization algorithm is continuous, so the parameters of $\mathrm{X}$ and $\mathrm{Y}$ are transmitted to the interval between zero and one. In order to transfer data from a discrete interval to a continuous, the equation (3) is used [23].

Value $_{\text {new }}=\frac{\text { Value }_{\text {old }}-\text { min }}{\text { max }- \text { min }}$

The structure of the particle optimization algorithm is then used to determine the starting points of the K-means algorithm. 


\section{2-2-1 Particle Structure}

Particles represent the starting points of the K-means clustering center. The number of points in the K-means algorithm is as large as the number of clusters. If the algorithm has two clusters, then two start points are required, and if the $\mathrm{K}$ algorithm is a cluster, then $\mathrm{K}$ is the starting point. Given that the data has 2 features, each point has 8 values between 0 and 1. Therefore, considering that clustering has a $\mathrm{K}$ point and each point has 2 values and its value is between 0 and 1 , this structure is shown in Fig. 1.

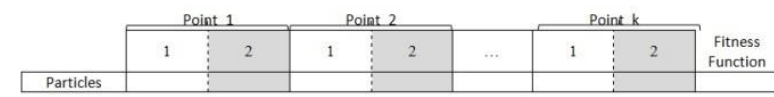 \\ Figure 1 Structure of proposed particle of the first stage}

\section{2-2-2 Production of initial population}

First, 20 particles are created and they are randomly range from 0 to 1 . For the production of the initial population, a uniform distribution is used, and in (4) the manner in which a uniform distribution between numbers 0 and 1 is shown.

$$
\text { Chromosome }=\mathrm{U}(0,1)
$$

\section{2-3 Calculation competency function}

In calculating the competency function this is how each particle is proportional to a K-means clustering. Each particle is the starting point of the K-means algorithm. Then the K-means algorithm is executed. After the K-means algorithm converges, the accuracy of clustering as a competency function will be sent.

Given that each particle is fully clustered, the result of clustering is calculated by the clustering accuracy parameter and its output is considered as the particle computation suitability. The accuracy of the Davies-Bouldin Index is used to calculate the accuracy [17].

The Davis-Bouldin method is a function of the total ratio of intra cluster dispersion to the distance between clusters. The Davis-Bouldin Validation Index is shown by equation (5), the Davis-Bouldin Method operates on the basis of minimization.

$D B=\frac{1}{n} \sum_{\substack{i=1 \\ i \neq j}}^{n} \max \left\{\frac{S_{n}\left(Q_{i}\right)+S_{n}\left(Q_{j}\right)}{S\left(Q_{i}, Q_{j}\right)}\right\}$

In which $\mathrm{n}$ is the number of clusters, $\mathrm{Sn}$ is the mean of the cluster data spacing from the cluster center and $\mathrm{S}(\mathrm{Qi}, \mathrm{Qj})$ is the distance between the centers of the clusters; therefore, when they are within the cluster and clusters are far from each other, this ratio gets small. The small amount of the Davis-Bouldin validation index is valid clustering representation. In Fig. 2, the structure of the calculation of the competency function is shown.

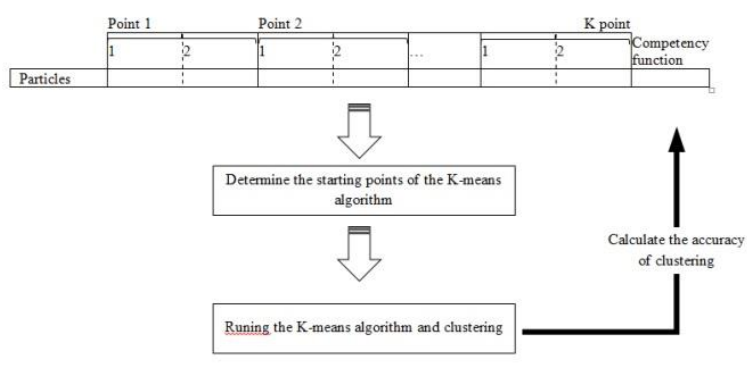

Figure 2 the structure of the proposed competency function

\section{2-2-4 Speed control}

One of the important aspects for determining the efficiency and accuracy of an optimization algorithm is how to reconcile Explore and Exploit with the proposed algorithm.

The Explore feature is the ability to search an algorithm in different areas of the search space to find optimal amount. On the other hand, the Exploit feature is the ability to focus the search around a likely area to improve the candidate's solution. Thus, we created an appropriate solution between these two conflicting goals, which is achieved by speeding up the PSO, as shown in equation (6).

$$
\begin{gathered}
v_{\mathrm{ij}}(\mathrm{t}+1)=\mathrm{v}_{\mathrm{ij}}(\mathrm{t})+\mathrm{c}_{1} \mathrm{r}_{1 \mathrm{j}}(\mathrm{t})\left[\mathrm{y}_{\mathrm{ij}}(\mathrm{t})-\mathrm{x}_{\mathrm{ij}}(\mathrm{t})\right] \\
+\mathrm{c}_{2} \mathrm{r}_{2 \mathrm{j}}(\mathrm{t})\left[\tilde{y}_{j}(\mathrm{t})-\mathrm{x}_{\mathrm{ij}}(\mathrm{t})\right] \\
v_{i j}(\mathrm{t}+1)= \begin{cases}v_{i j}(t+1) & \text { if } v_{i j}(t+1)<v_{\max , j} \\
v_{\text {max }, j} & \text { if } v_{i j}(t+1) \geq v_{\max , j}\end{cases}
\end{gathered}
$$

In which $v_{\text {max }, j}$ is the highest speed in the number of tables and the number of columns, the value of $v_{\text {max }, j}$ is very important. Because it speeds up search through inhibition. If the value is large. The Explore feature will increase the algorithm. While the small values of this parameter, the local Exploit feature improves the algorithm. If $\mathrm{v}_{\text {max,j }}$ is too small, congestion may not be as good as local areas. In addition, there is the possibility of clustering in the optimal local area, which will not be able to get out of it for the algorithm. On the other hand, the large amounts of $v_{\text {max,j }}$ have the risk of losing good areas. Particles may jump through good solutions and search for inappropriate areas. Large values result in the algorithm moving away from the optimal area. In this case, the particles move faster.

Finding the right amount of $\mathrm{v}_{\text {max }, \mathrm{j}}$, in order to establish two types of equilibrium as follows:

- Fast or slow motion

- $\quad$ Explore and Exploit

Chromosome $=\mathrm{U}(0,1)$

In which $x_{\mathrm{max}, \mathrm{j}}$ and $x_{\mathrm{min}, \mathrm{j}}$, respectively, are the minimum and maximum value of the table number and the column number in both dimensions, and $\delta \in(0,1]$ the $\delta$ value is initially equal to one, and in each generation, the $\delta$ value of (8) changes. The amount of $\delta$ each generation is $90 \%$ less than the previous generation.

$\delta=0.9^{i}, \mathrm{i}=$ generation number 


\subsubsection{The condition for stopping the algorithm}

The condition to end the algorithm is based on the congestion radius. The criterion for the end of the algorithm is close to zeroing the normalized congestion radius. We calculated the normalized congestion radius from (9).

$R_{\text {norm }}=\frac{R_{\max }}{\text { diameter }(S)}$

In which diameter(S) is the diameter of the space in the initial congestion and $\mathrm{R}_{\max }$ is the maximum radius, calculated from equation (10).

$R_{\text {max }}=\left\|x_{m}-\hat{y}\right\| \quad, m=1, \ldots, n_{s}$

When $R_{n o r m}$ is closer to zero, the algorithm stops, $\hat{y}$ is the minimum value of the competency function.

\section{2-2-6 The structure of the particle optimization algorithm}

It includes the following six steps:

Step 1: Initialize the parameters of the velocity and location of the particles in the initial crowd according to (4)

Step 2: Updating the best local position of particle i for all particles

Step 3: Improving the best global position of all particles

Step 4: Calculating the new velocity of all particles by means of equation (6)

Step 5: Calculating the new location of all particles by means of equation (11)

$x_{i}(t+1)=x_{i}(t)+v_{i}(t+1)$

Step 6: Repeating steps 2 to 5 with the output condition of the algorithm according to 2-2-5.

\section{2-3 Optimization of Particle Algorithm Using Genetic and Heuristic Algorithm}

In the sequel, every generation of the genetic algorithm runs. Using the genetic algorithm increases the search power of the particle optimization algorithm.

\section{2-3-1 Initial population}

In the previous step, 20 particles are generated that identify the starting points of the K-means algorithm, thus, K-means algorithm is applied to each particle, and the cluster output is considered as the input of the mantle algorithm. The fragmental particle structure of particle optimization algorithm for the chromosome is shown in Fig. 3.

\section{2-3-2 Combinations}

In the study, three methods of One-point Crossover, Two-point Crossover and uniform-point Crossover were used.

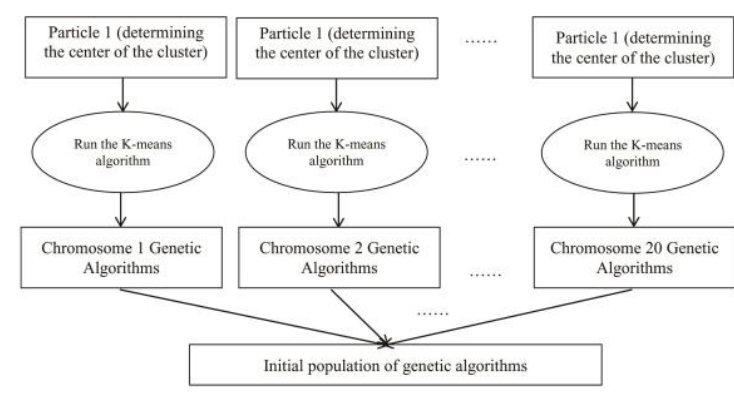

Figure 3 Particle conversion of particle optimization to the initial population of the algorithm

In the single-point Crossover, first, a random point is selected in the sequence of the parent chromosomes, and then from the selected location, the chromosome of both parents is cut. The first part of the first parent and the second part of the second parent are used to produce the first child. The second child consists of the first part of the second parent and the second parent of the first (Figure 4).

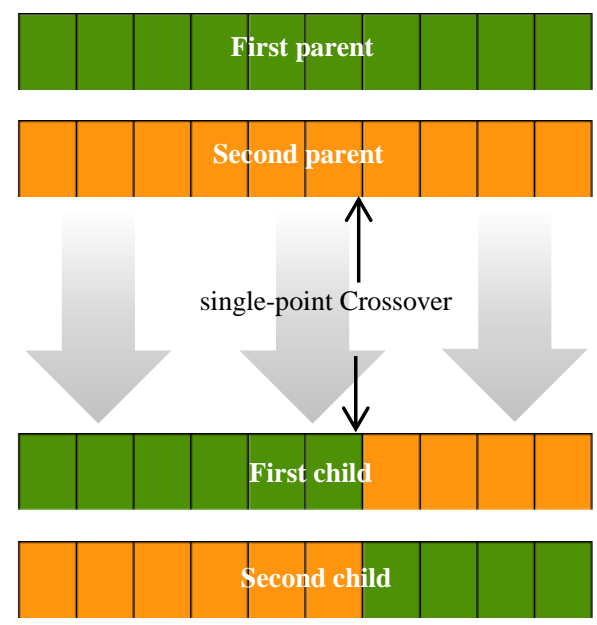

Figure 4 single-point crossover

In the two-point crossover, two random points are selected in the parental chromosome sequence, and then the parent chromosome is cut off from these points. The first and third parts of the first parent and the second part of the second parent are used to produce the first child. The second child consists of the first and third parts of the second parent and the second part of the first parent's department (Fig. 5). Obviously, by increasing the number of breakpoints in the multi-point multiplication operator, the similarity of the parent to each parent decreases and the reciprocity function divergence will be strengthened.

In the uniform combination operator, the value of the child's gene is selected according to the values of the corresponding genes of both parents. In this method, the genes of each parent have equal chances for the presence in the corresponding genes of the child. In the uniform reconciliation operator, it is determined by the random distribution that the amount of each child's gene is selected from the corresponding gene value of the parent (Fig. 6). 


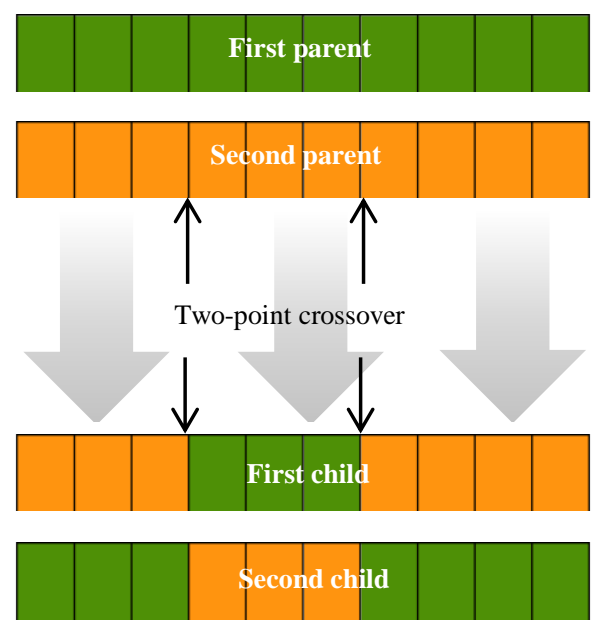

Figure 5 Two-point crossover

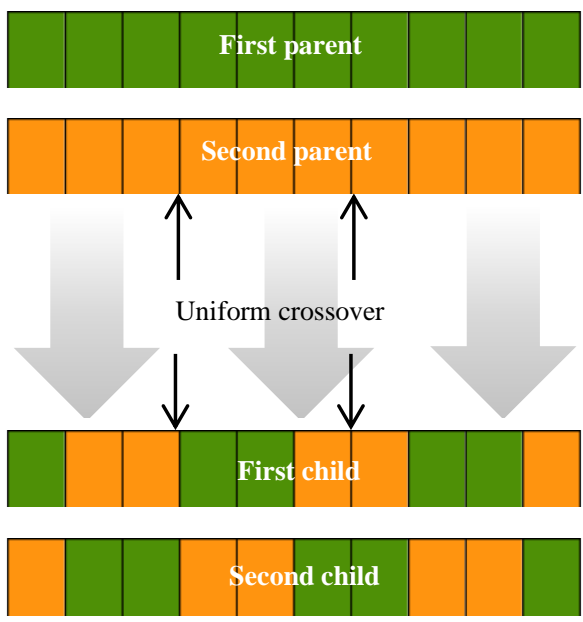

Figure 6 Uniform crossover

In this research, three crossovers are used. In the first $50 \%$ of the genetic algorithm, the uniform crossovers algorithm is used because the uniform composition has a high divergence, and from $50 \%$ to $75 \%$ of the genetic algorithm, the two-point algorithm is used, and in $25 \%$ of end of the algorithm a singlepoint crossover is used. The composition structure of the combination in the genetic algorithm is shown in Fig 7.

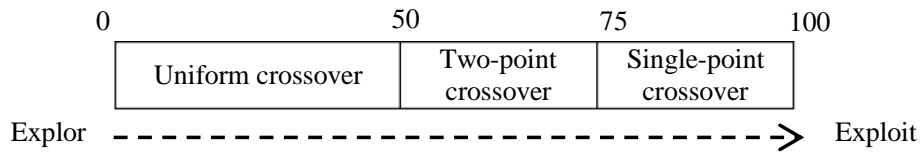

Figure 7 Crossover execution structure

\section{2-3-3 Mutation}

The mutation is the random change process of the amount of genes in a chromosome, the main goal in the mutation operator is to find new values for the genes of the offspring (values not found in none of the parents) to increase genotype variation in the population. The mutation should be performed so that the genes do not deteriorate in superior responses. Given that the mutation operator causes a random change in the chromosome, the use of this operator increases the probability of finding new values for the genes. Using more than this operator increases the divergence of the algorithm.

In this research, the contents of a part of a chromosome are selected and re-established. In Fig. 8, the structure of the mutation is observed.

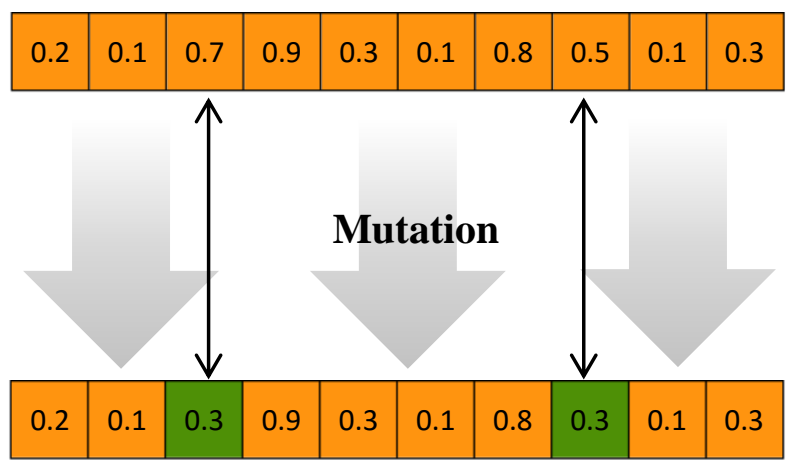

Figure 8 mutation structure

A generational replacement method is used, because this method has the convergence and divergence capability by changing its variables. Initially, the algorithm transfers $50 \%$ of the parent and $50 \%$ of the children to the next generation, causing a divergence in the problem. After each generation, the percentage of children decreases and the percentage of parents increases to bring about convergence. The percentage of parents' increase is calculated through trial and error.

\section{2-3-5 Condition of ending the algorithm condition}

In this research, the condition for ending of the genetic algorithm is to execute 100 generations of genetic algorithms unchanged.

\section{EVALUATION \\ OF PROPOSED ALGORITHM}

Testing and proving their results is one of the most important parts of a model is. We have developed programs in the language of mathematics to evaluate the proposed model, which we will continue to explain and illustrate the results obtained from them. These tests were performed on the Windows 7 operating system and a 5-core computer with 2.7GH processors and 4GB of memory. The Jaguar standard was used to test this algorithm.

In the evaluation, the proposed algorithm was evaluated using the following four methods.

- K-Means

- K-Medoids

- Hierarchical Clustering

- ANN

The parameters of the proposed algorithm are shown in Table1. These parameters are calculated with trial and error. 
Table 1 Parameters of the proposed algorithm

\begin{tabular}{|l|l|}
\hline Parameter & Value \\
\hline $\begin{array}{l}\text { Initial population of particle optimization } \\
\text { algorithm }\end{array}$ & 100 \\
\hline $\mathrm{C}_{1}$ & 2 \\
\hline $\mathrm{C}_{2}$ & 2 \\
\hline Initial population of genetic algorithms & 100 \\
\hline Combining Genetic Algorithms & 0.7 \\
\hline Genetic Algorithm Mutation & 0.3 \\
\hline Number of Generations of Genetic Algorithms & 100 unchanged generations \\
\hline
\end{tabular}

The proposed algorithm was implemented with different clusters, and its output is shown in Table 2.

Table 2 Comparing 5 methods of solving bank clustering problem with Davis-Bouldin Index of accuracy

\begin{tabular}{|c|c|c|c|c|c|}
\hline & K-Means & K-Medoids & $\begin{array}{c}\text { Hierarchical } \\
\text { Clustering }\end{array}$ & ANN & $\begin{array}{l}\text { Proposed } \\
\text { algorithm }\end{array}$ \\
\hline Cluster 2 & 0.74 & 0.53 & 0.78 & 0.78 & 0.61 \\
\hline Cluster 3 & 0.59 & 0.49 & 0.64 & 0.64 & 0.31 \\
\hline Cluster 4 & 0.7 & 0.54 & 0.61 & 0.93 & 0.23 \\
\hline Cluster 5 & 0.84 & 0.53 & 0.59 & 0.85 & 0.18 \\
\hline Cluster 6 & 0.82 & 0.52 & 0.52 & 1.01 & 0.18 \\
\hline Cluster 7 & 0.9 & 0.49 & 0.58 & 1.01 & 0.13 \\
\hline Cluster 8 & 0.92 & 0.48 & 0.52 & 1.01 & 0.11 \\
\hline Cluster 9 & 0.92 & 0.53 & 0.5 & 1.05 & 0.14 \\
\hline $\begin{array}{c}\text { Cluster } \\
10 \\
\end{array}$ & 0.95 & 0.53 & 0.47 & 1.05 & 0.14 \\
\hline $\begin{array}{c}\text { Cluster } \\
11\end{array}$ & 0.93 & 0.53 & 0.44 & 1.01 & 0.15 \\
\hline $\begin{array}{c}\text { Cluster } \\
12\end{array}$ & 0.9 & 0.53 & 0.44 & 0.97 & 0.16 \\
\hline
\end{tabular}

As in Table 2, the proposed algorithm works better than other methods. Figure 9 shows the comparison of the best output of each algorithm. As shown in Fig. 9, the proposed algorithm works better than other methods.

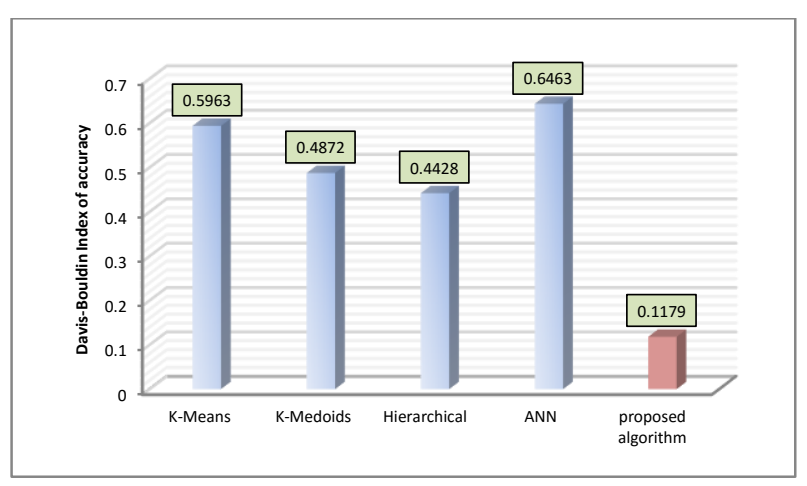

Figure 9 Comparison of proposed algorithm with 4 other algorithms with Davis-Bouldin Index of accuracy

\section{CONCLUSIONS AND FUTURE STUDIES}

Choosing location is one of the important factors in economic enterprises activities. Due to this importance, location-based science has also sought to provide methods and techniques for determining the location of activities of firms. Banking as an economic activity seeks to use scientific methods to maximize service coverage and efficiency and minimize costs. ATM machines as an electronic technology have been part of this goal in recent years.

In this research, we tried to present a complete overview of the clustering issue of a more than a decade of activities in this field. It also tried to outline some of the applied methods and their strengths and weaknesses. But what is most noticed in the most successful work of the past was the application of high level heuristic and a high-level perception that have attracted many researchers to this field.

In this study, the combination of three K-means clustering algorithms, genetic algorithm and particle optimization algorithm were used to solve the problem. Also, the way to create a database and location of ATM to assess the clustering problem of ATMs.

In this study, the combination of three K-means clustering algorithms, genetic algorithm and particle optimization algorithm have been shown to be of great power in solving optimization problems. It was also found that the implementation of this algorithm was very time-consuming but had an appropriate output.

As stated, intrusion detection is one of the important issues in the field of security in computer networks and all issues related to it can be the subject of research of numerous articles and dissertations. The following topics are suggested for future work:

Now, in order to proceed with the clustering work, using the optimization algorithm, here are some suggestions for following studies:

1. More advanced mutations and combinations and fuzzy mutations can be used.

2. The K-means algorithm can be used instead of the Kmedoids algorithm.

3. Particle optimization algorithm can be used to increase the speed of the algorithm.

4. Other algorithms can be used instead of ant colony instead of particle optimization algorithm.

5. Fuzzy logic can be used instead of tuning the parameters of the problem in trial and error method.

\section{REFERENCES}

[1] Goli, Olfat, Liaia, and Fuquardi, "Locating ATMs Using Analytical Hierarchy Process (AHP) Case Study: Branches of Keshavarzi Bank of Tehran 10th District," Geography and Development Quarterly

[2] L. Zhao, B. Garner, and B. Parolin, "Branch bank closures in Sydney: A geographical perspective and analysis," in 12th International Conference on Geoformatics, Sweden, 2014.

[3] N. Al-Hanbali and others, "Building a Geospatial database and GIS data-Model integration for Banking: ATM site location," in Commission IV Joint Workshop: Data Integration and Digital Mapping Challenges in Geospatial Analysis, Integration and Visulization II, Stuttgart, Germany, September8-9, 2013. 
[4] M. A. Aldajani and H. K. Alfares, "Location of banking automatic teller machines based on convolution," Comput. Ind. Eng., vol. 57, no. 4, pp. 1194-1201, 2016.

[5] H. F. Sabokbar, G. H. Ashournejad, S. Rahimi, and A. Farhadipoor, "Assessing the potential number of ATMs in banks, financial and credit institutions using Analytic Network Process (ANP) and Gray Clustering Analysis (GCA) Case study: between Enghelab Sq and Ferdowsi Sq-Enghelab Street of Tehran," Reg. Urban Stud. Res., vol. 4, no. 14, pp. 23-42, 2012.

[6] M. Almossawi, "Bank selection criteria employed by college students in Bahrain: an empirical analysis," Int. J. Bank Mark., vol. 19, no. 3, pp. 115-125, 2014.

[7] L. Bach, "Locational models for systems of private and public facilities based on concepts of accessibility and access opportunity," Environ. Plan. A, vol. 12, no. 3, pp. 301-320, 2001.

[8] C. Jensen, Data Mining: Beginners' Analytics Guide for Business and Science. CreateSpace Independent Publishing Platform, 2017.

[9] I. H. Witten, E. Frank, M. A. Hall, and C. J. Pal, Data Mining, Fourth Edition: Practical Machine Learning Tools and Techniques, 4 edition. Amsterdam: Morgan Kaufmann, 2016.

[10] I. H. Witten, E. Frank, and M. A. Hall, Data Mining: Practical Machine Learning Tools and Techniques, Third Edition, 3 edition. Burlington, MA: Morgan Kaufmann, 2011.

[11] M. J. Zaki and W. M. Jr, Data Mining and Analysis: Fundamental Concepts and Algorithms, 1 edition. New York, NY: Cambridge University Press, 2014.

[12] N. Aggarwal and K. Aggarwal, An Improved K-means Clustering Algorithm For Data Mining. S.l.: LAP LAMBERT Academic Publishing, 2012.

[13] M. E. Celebi, Ed., Partitional Clustering Algorithms, 2015 edition. New York: Springer, 2014.

[14] A. Yerpude and S. Dubey, Modified K- Medoids Algorithm For Image Segmentation: Application of Clustering in Image Processing. Saarbrücken: LAP LAMBERT Academic Publishing, 2012.

[15] S. M. Savaresi and D. L. Boley, "On the performance of bisecting K-means and PDDP," in Proceedings of the 2001 SIAM International Conference on Data Mining, 2001, pp. 1-14.

[16] M. E. Karim, Fuzzy C-means Clustering using Pattern Recognition: Concepts, Methods, Implementations. LAP LAMBERT Academic Publishing, 2011.

[17] M. E. Celebi, Ed., Partitional Clustering Algorithms, 2015 edition. New York: Springer, 2014.

[18] G. C. Calafiore and L. E. Ghaoui, Optimization Models, 1 edition. Cambridge: Cambridge University Press, 2014.
[19] R. K. Arora, Optimization: Algorithms and Applications, 1 edition. Boca Raton: Chapman and Hall/CRC, 2015.

[20] A. Rathi, Optimization of Particle Swarm Optimization Algorithm. Saarbrücken: LAP LAMBERT Academic Publishing, 2013.

[21] K. E. Parsopoulos and M. N. Vrahatis, Particle Swarm Optimization and Intelligence: Advances and Applications, 1 edition. Hershey, PA: IGI Global, 2010.

[22] M. Morzy, "Prediction of moving object location based on frequent trajectories," in Computer and Information Sciences-ISCIS 2006, Springer, 2006, pp. 583-592.

[23] J. Han, M. Kamber, and J. Pei, Data Mining: Concepts and Techniques, Third Edition, 3 edition. Haryana, India; Burlington, MA: Morgan Kaufmann, 2011.

[24] M. Mitchell, An Introduction to Genetic Algorithms, Reprint edition. Cambridge, Mass.: MIT Press, 1998. 